\title{
S.-T. Yau Mathematics Awards
}

\section{by Thomas Kwok-Keung Au, Sen Hu, Hao Xu, and Hong-Wei Xu}

\author{
Chinese University of Hong Kong \\ University of Science and Technology of China \\ Harvard University \\ Zhejiang University
}

\section{S.-T. Yau College Mathematics Contests}

A mathematics contest for college students every year organized by Shing-Tung Yau by is started from 2010.

On the suggestion of Shing-Tung Yau it is proposed to organize college student mathematics contests. By competing with their peers it would help to enhance undergraduates in their basic college training in mathematics. The examination shall cover wide range of subjects. With deep and interesting questions this would motivate stu-

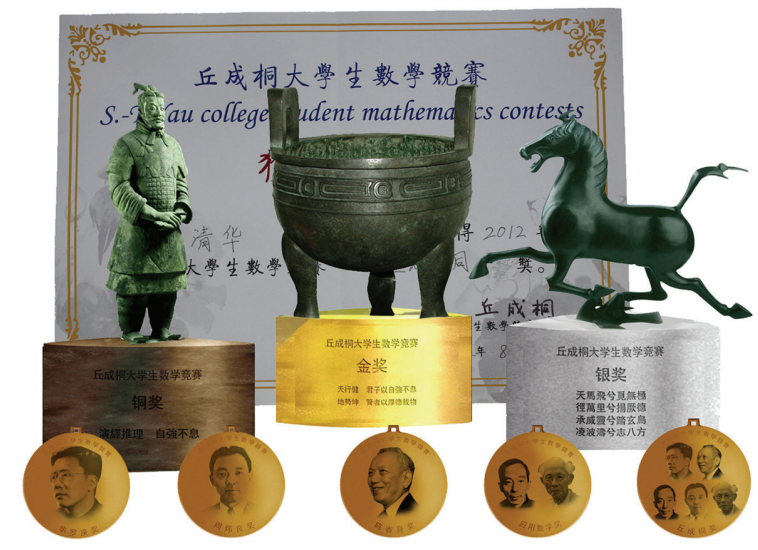

S.-T. Yau Mathematics Awards

dents to learn. There shall be an outstanding panel to oversee it.

Young talents in China are abundant. The aim of the competition is to help many students in China to acquire basic trainings in modern mathematics. It is a basic step to build up a solid foundation in the developments of mathematics in China. We believe this will bring college mathematics education in China to a higher level and it would help to transform a country of vast population into a country of vast human resources. The test results shall be important references to admitting graduate students both in and outside of China.

The competition is open only to regularly enrolled undergraduates, in colleges and universities of mainland China, Hong Kong and Taiwan, who have not yet received a college degree. The contests are on 5 subjects:

1. Analysis and Differential Equations

2. Geometry and Topology

3. Algebra, Number Theory

4. Applied and Computational Mathematics

5. Probability and Statistics

A college or university with at least five registered entrants obtains a team rank. One team consists of five individual contestants. There will be contests for teams and for individuals separately. In the first morning there will be the team contest. In the team contest, team members shall discuss to divide problems among themselves in the first half hour. Then each team member works independently on the problems in the remaining two and half hours. There will be individual contest in the remaining one and half days. Contest for each subject shall be last for two and half hours. No collaboration or outside assistance is permitted during the examination for individual contests and for team contests.

Below is an open letter from Prof. Yau:

\section{Dear Colleagues,}

We are initiating a College Student Mathematics Contest. In this letter I shall explain more clearly the needs of this college mathematics competition. We wish the competition helps to achieve the following goals.

1. In the training of a good mathematician a crucial step is to have students to know basic subjects and skills in mathematics before they do research. To achieve this major universities in the world set up 
qualify exams for beginning graduate students. Good students majoring in mathematics in Chinese universities should be good enough to pass qualify exams in any major universities in the world, e.g. Harvard, Stanford, Berkeley, Princeton, Columbia, etc. However in US we are facing the problem that some good Chinese students could not pass while good students from other countries could, especially those from Eastern European countries. The College Mathematics Contest shall be broad enough to cover the subjects in those qualify exams and it shall be helpful to train many good students in mathematics

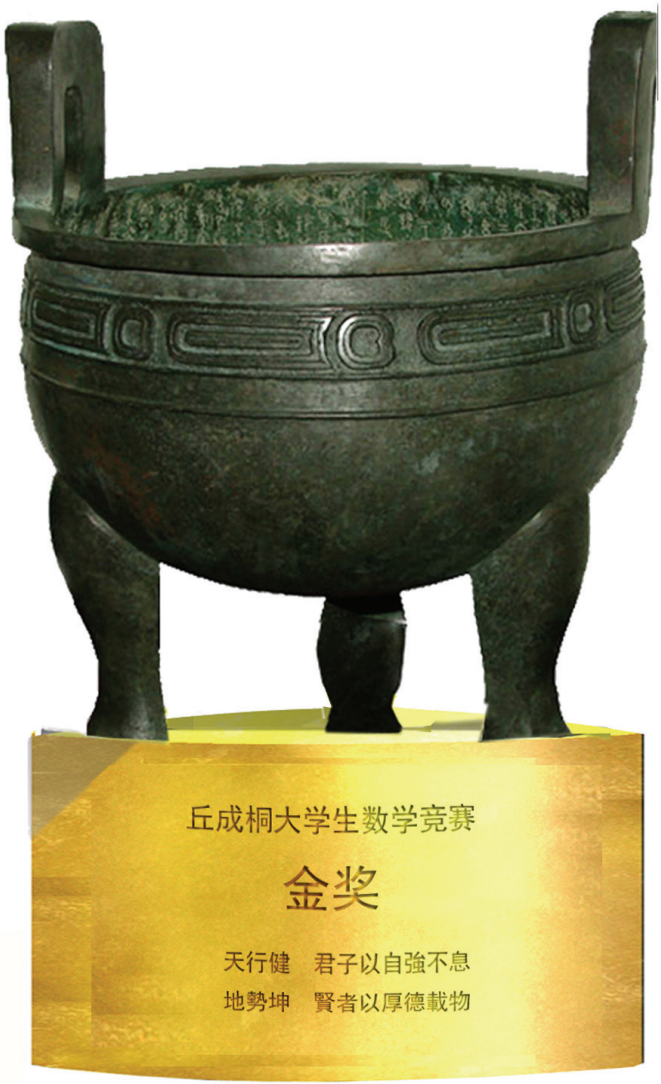

The Trophy of the S.-T. Yau Mathematics Gold Award

for China and for the world.

2. It should be a fair competition so that students from "second rated" universities in China are given a second chance to compete. This is very important. There are many reasons that a high school student did not get into top universities such as bad luck, poor health, poverty, or immature. But it is often the case that situations changed when they go to college, they become more mature and suddenly they decide to be good. With the present situation, students of such colleges have hard time to go to the best universities to do graduate study. We should give them a chance.
After all, when I was educated in Hong Kong, it was difficult for me to go to the first rated universities. It was through my hard work and good luck that I became recognized.

3. To make sure those top university students to know that there are continuous challenges for their mathematics life so that they have to work hard at a fundamental level. It is very popular to see those students from college in China to talk about philosophy of mathematics while they have no idea on how to sit down to do a mathematics computation, this can be changed most effectively by such a competition.

4. We shall invite world class mathematicians to give students oral test (for the top 15 candidates) and their names will endorse the quality of those students and it will be helpful for them to go to the best graduate schools. I am sure that many mathematicians would like to meet those brilliant students. In short I believe that the competition will bring the ability of our students to a much higher level. We welcome your students to participate the College Mathematics Contest.

With best regards,

Shing-Tung Yau

Chair of the Scientific and Questions Committee of S.-T. Yau College Mathematics Contests

\section{S.-T. Yau College Mathematics Contests Winners Finalists}

\section{K. Ниа Medals (Analysis and Differential Equations)}

Gold: Weiluo Ren (USTC)

Silver: Teng Fei (Tsinghua), Chen He (Zhejiang University), Borui Liu (USTC)

Bronze: Weijie Su (Peking University), Qing Xu (Fudan), Xiaoyu Zhang (Zhejiang University), Shi Gu (Tsinghua), Tianyu Wu (Tsinghua), Zijun Zhou (Tsinghua)

\section{S. S. Chern Medals (Geometry and Topology)}

Gold: Yunqing Tang (Peking University)

Silver: Zhuohui Zhang (Zhejiang University), Yi Xie (Zhejiang University), Hua Qiang (Peking University)

Bronze: Ruixiang Zhang (Peking University), Zijun Zhou (Tsinghua), Weijie Yang (CUHK), Chen He (Zhejiang University), Zhiyuan Zhang (Peking University), Hua Qiang (Peking University)

\section{W. L. Chow Medals (Algebra, Number Theory and Combinatorics)}

Gold: Teng Fei (Tsinghua)

Silver: Youwei Fan (National Taiwan University), Yunqing Tang (Peking University), Yi Xie (Zhejiang University) 
Bronze: Honglu Fan (Zhejiang University), Zhuohui Zhang (Zhejiang University), Zhijie Huang (Tsinghua), Daxin $\mathrm{Xu}$ (Peking University), Weijie Su (Peking University), Chen He (Zhejiang University)

\section{P. L. Hsu-C. C. Lin Medals (Applied Math., Computational} Math., Probability and Statistics)

Gold: Weijie Su (Peking University)

Silver: Xin Sun (Peking University)

Bronze: Qingyuan Zhao (USTC), Borui Liu (USTC)

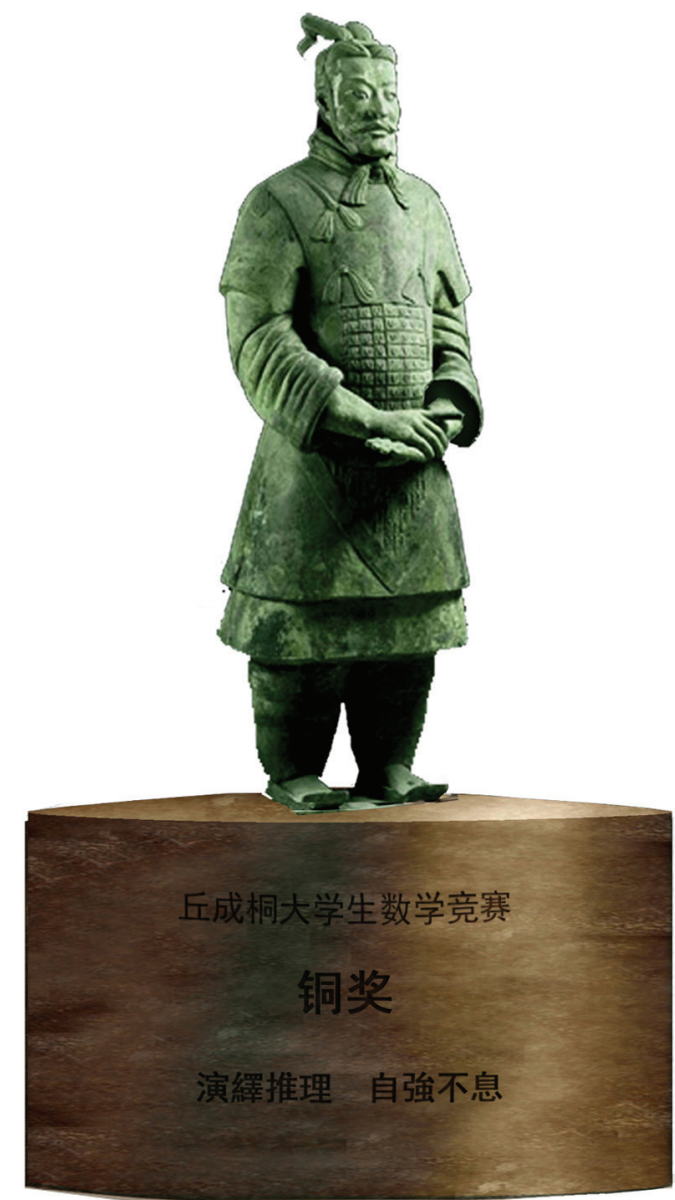

The Trophy of the S.-T. Yau Mathematics Bronze Award

\section{S.-T. Yau Medals (Individual Overall)}

Gold: Weijie Su (Peking University), Teng Fei (Tsinghua), Yunqing Tang (Peking University)

Silver: Chen He (Zhejiang University), Yi Xie (Zhejiang University)

Bronze: Borui Liu (USTC), Weiluo Ren (USTC), Daxin Xu (Peking University), Zhuohui Zhang (Zhejiang University), Zijun Zhou (Tsinghua)
The S.-T. Yau College Mathematics Contests Team Winners

Gold: (Tsinghua) Teng Fei, Zhijie Huang, Zijun Zhou, Shi $\mathrm{Gu}$

Silver: (Peking University) Daxin Xu, Chenjie Fan, Zhiyuan Zhang, Ruixiang Zhang

Bronze: (Tsinghua) Duanyang Zhang, Tianyu Wu, Yalong Cao, Xin yang (USTC) Borui Liu, Weiluo Ren, ZYimin Zhong, Dong Xia (Wuhan University) Xueying Yu, Yu Yang, Hui Kan, Huaying Fang

\section{S.-T. Yau College Mathematics Contests Winners Finalists}

\section{K. Hua Medals (Analysis and Differential Equations)}

Gold: Chenjie Fan (Peking University)

Silver: Ruofan Wang (Tsinghua), Ruixiang Zhang (Peking University), Boyu Zhang (Peking University)

Bronze: Xiaoyu Zhang (Zhejiang University), Gao Chen (USTC), Yuan-Jun Gao (Tsinghua), Bin Gui (Shanghai Jiaotong University), Lizao Ye (Peking University), Songyan Xie (Tsinghua)

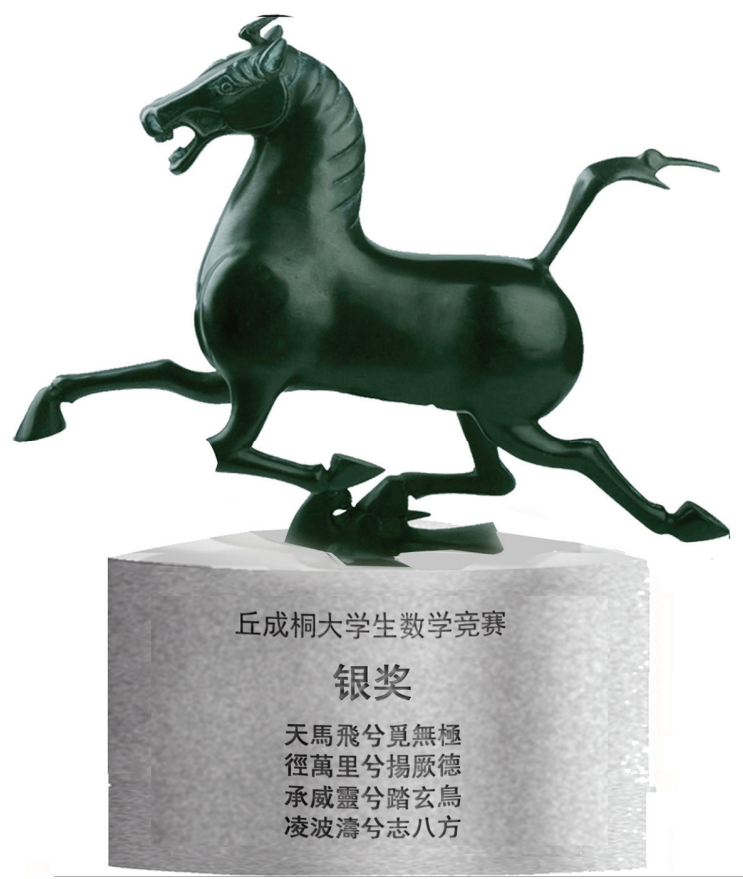

The Trophy of the S.-T. Yau Mathematics Silver Award

\section{S. S. Chern Medals (Geometry and Topology)}

Gold: Lizao Ye (Peking University)

Silver: Chao Xu (Zhejiang University), Chenglong Yu

(Tsinghua), Ruixiang Zhang (Peking University), Boyu Zhang (Peking University)

Bronze: Honglu Fan (Zhejiang University), Yi-Hang Zhu (Tsinghua), Chen-Jie Fan (Peking University), Bin Gui (Shanghai Jiaotong University), Tao Su (Tsinghua) 


\section{W. L. Chow Medal (Algebra and Number Theory)}

Gold: Zhuo Chen (Peking University)

Silver: Yang Zhou (Zhejiang University), Yihang Zhu (Tsinghua), Ruixiang Zhang (Peking University)

Bronze: Hanlong Fang (Tsinghua), Jun Su (Peking University), Shu-Ting Huang (National Taiwan University), Chenjie Fan (Peking University), Gao Chen (USTC), Tao Su (Tsinghua)

\section{P. L. Hsu-C. C. Lin Medal (Applied Mathematics,} Probability and Statistics)

Gold: Xufei Wang (Tsinghua)

Silver: Haifeng Xu (USTC), Simeng Kuang (Peking University), Gao Chen (USTC)

Bronze: Jie-chao Xiong (Peking University), Pei-chao Peng (Peking University), Jun Gao (Tsinghua), Run-Dong Du (USTC), Yuan-Jun Gao (Tsinghua)

\section{S.-T. Yau Medals (Individual Overall)}

Gold: Ruixiang Zhang (Peking University), Boyu Zhang (Peking University)

Silver: Lizao Ye (Peking University), Gao Chen (USTC), Chenjie Fan (Peking University), Ruofan Wang (Tsinghua), Zhuo Chen (Peking University)

Bronze: Tao Su (Tsinghua), Xu-fei Wang (Tsinghua), Yi-hang Zhu (Tsinghua)

The S.-T. Yau College Mathematics Contests Team Winners:

Gold: (Peking University) Chenjie Fan, Simeng Kuang,Lizao Ye,Ruixiang Zhang

Silver: (Tsinghua) Jun Gao,Tao Su,Songyan Xie,Yihang Zhu Bronze: (Tsinghua)Wei Chai, Hanlong Fang, Jiayu Peng, Xufei Wang (USTC) Gao Chen, Yiqiu Mao, Haifeng Xu, Minghan Yan

\section{S.-T. Yau College Mathematics Contests Winners Finalists}

\section{K. Hua Medals (Analysis and Differential Equations)}

Gold: Yubo Zhang (Peking University)

Silver: Yupeng Le (Tsinghua), Zonghan Sun (Tsinghua), Ziliang Che (Tsinghua)

Bronze: Zicheng Qian (USTC), Jin Gong (Wuhan University), Yingdi Qin (Fudan)

\section{S. S. Chern Medals (Geometry and Topology)}

Gold: Boyu Zhang (Peking University)

Silver: Yang Zhou (Zhejiang University), Chenglong Yu (Tsinghua)

Bronze: Yan Li (Zhejiang University), Zhengyi Zhou (Nanjing University), Yiwen Zhou (Peking University)

\section{W. L. Chow Medal (Algebra and Number Theory)}

Gold: Dongyi Wei (Peking University)

Silver: Lüe Pan (Peking University), Yang Zhou (Zhejiang University)

Bronze: Jun Su (Peking University), Ziquan Zhuang (Peking University), Chenglong Yu (Tsinghua)

\section{C. Lin Medal (Applied Mathematics)}

Gold: Dongyi Wei (Peking University)

Silver: Jiajun Tong (Peking University), Xianglong Duan (USTC)

Bronze: Ying liu (Fudan), Mingyue Li (USTC), RouxinWang (National Taiwan University)

\section{P. L. Hsu Medal (Probability and Statistics)}

Gold: Xinran Li (Peking University)

Silver: Ziliang Che (Tsinghua), Yu Lu (Peking University)

Bronze: Meilei Jiang (Tsinghua), Zhiwei Zheng (Tsinghua), Xianglong Duan (USTC), Dongyi Wei (Peking University)

\section{S.-T. Yau Medals (Individual Overall)}

Gold: Boyu Zhang (Peking University), Chenglong Yu (Tsinghua)

Silver: Dongyi Wei (Peking University), Yang Zhou (Zhejiang University)

The S.-T. Yau College Mathematics Contests Team Winners:

Gold: (Peking University) Siqi He, Yiwen Zhou, Zhiyuan Ding, Jiajun Tong, Xinran Li

Silver: (USTC) HuaWang, Zheng Lu, Mao Li, Jianwei Xiao, Xing Lü (Peking University) Chao Li, Lüe Pan, Bo Lin, $\mathrm{Ze} \mathrm{Xu,} \mathrm{Yue} \mathrm{Wang} \mathrm{(Tsinghua)} \mathrm{Linyuan} \mathrm{Liu,} \mathrm{Pengyu} \mathrm{Le,}$ Chenglong Yu, Lang Mou, Ziliang Che

Bronze: (Tsinghua) Jingrui Cheng, Ming Zhang, Qirui Li, Hao Zheng, Meilei Jiang (Peking University) Yang Lan, Ziquan Su, Jun Su, Fan Yao, Huanran Lu

\section{S.-T. Yau High School Mathematics Awards}

Mathematical Sciences play a prominent and important role in the science and technology, as well as human resource competition, which are also gradually and widely applied in the science and technology in our daily life. We believe that youngsters should have excellent mathematical education in order to meet the challenges in the future. It was advocated early in the west that students should be fostered and encouraged to have the science research ability and therefore awards were set up. Siemens Westinghouse Science and Technology Competition, for example, is a special mathematics-related research award facing high school students. It focuses on innovation and practice, encourages team spirit and promotes the science research enthusiasm of high school students 
in the US. Many previous awardees later became famous scientists.

In 2004, Professor Shing-Tung Yau and Mr. Ronni Chan, Chairman of Hang Lung Group, founded the biannual "Hang Lung Mathematics Awards" for high school students in Hong Kong. It greatly stimulated the passion and dedication to science and technology of high school students and teachers in Hong Kong, and therefore produced a good social response.

Shing-Tung Yau initiated to hold a mathematics competition for high school students of Chinese decent around the world. He hopes to encourage the mathematical research and development, stimulated youngsters' interest in mathematics and most importantly cultivates Chinese mathematical talents from all over the world.

Mr. Dongsheng Chen, Chairman of Taikang Life Insurance Company Ltd., gave full support to Professor Yau's idea. Professor Yau and Mr. Chen have met many times in Beijing and Hangzhou and talked about the details for the set-up of an award for the secondary school students. Finally, it was agreed that the two parties cooperated and set up "S.-T. Yau High School Mathematics Awards."

In 2008, John Templeton Foundation decided to donate 1.2 Million USD to support S.-T. Yau High School Mathematics Awards for the first three years.

The Mathematics Awards will encourage innovation and teamwork spirit for Chinese high school students from all over the world. The previous award ceremonies

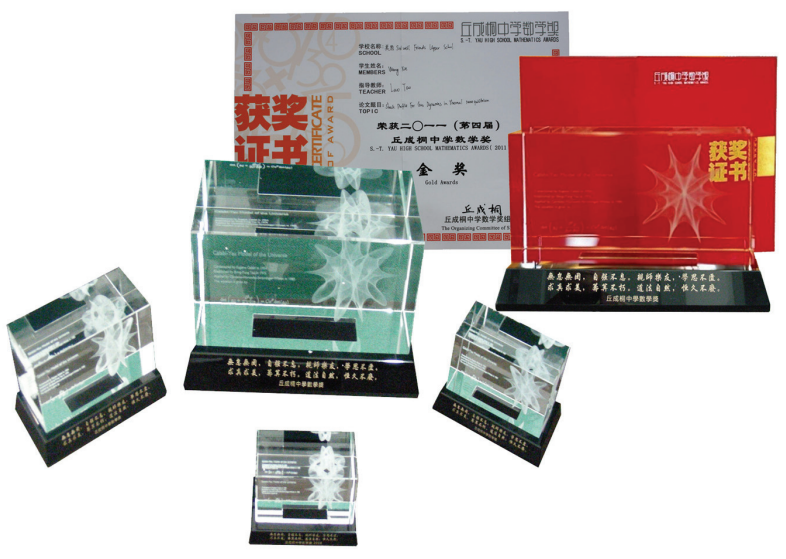

Certificates and Trophies of S.-T. Yau High School Mathematics Awards

had been held in Beijing (Oct. 2008, Dec, 2009, Dec. 2010, Dec. 2012) and Sanya (Dec. 2011). For the purpose of enhancing the interest and research capabilities of students in applied mathematical sciences, the Applied Mathematical Sciences Awards are initiated in 2010. Many awardees have been enrolled by international prestigious universities.
The final competition, 10 research teams of each award (Mathematics Award and Applied Mathematical Sciences Award) will get the competition award.

- One Gold Award for each award, 150,000RMB each

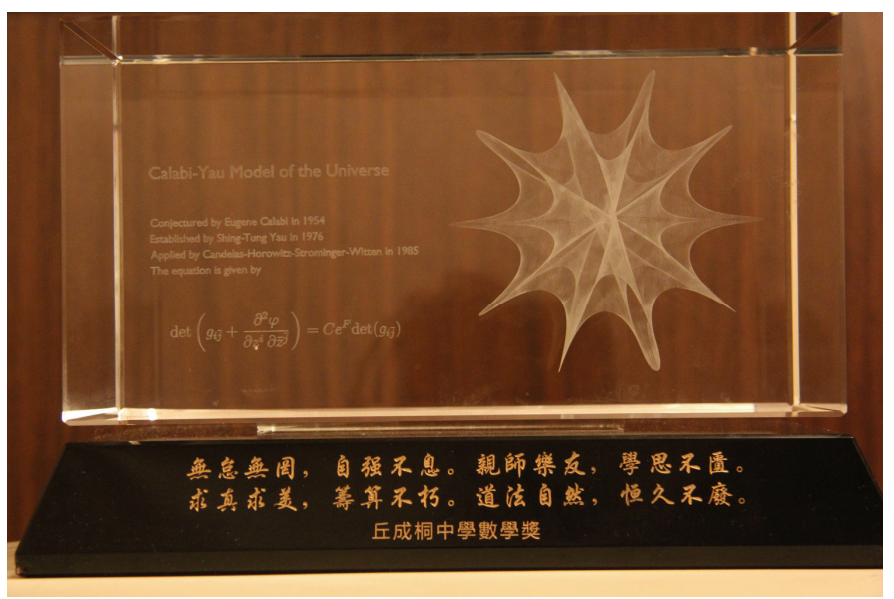

Trophy of S.-T. Yau High School Mathematics Gold Award

- One Silver Award for each award, 100,000RMB each

- Three Bronze Award for each award, 60,000RMB each

- Five Honorable Mention for each award, 30,000RMB each

The supervisor and the school each will be awarded $15 \%$ of the cash prize of the corresponding award. There will be a special award for research in financial math called the "Fellow of Actuaries."

\section{S.-T. Yau High School Mathematics Awards}

\section{Gold Award}

Topic: A research on the Minimum Prime Quadratic Residue Modulo a Prime

Members: Xiao Zhang, Lizao Ye, Weijun Fang

Teacher: Xiangyou Chen

School: Wenzhou High School

Silver Award

Topic: Invertibility Probability of Binary Matrices Members: Angela Dai, Caroline Kim, John Kim Teacher: Guofang Wei School: Dos Pueblos High School

\section{Bronze Award}

Topic 1: Optimized Methods of the Equalized Sprinkling Irrigation for Greenery Patches

Members: Yudi Fu, Zhoujia Li

Teacher: Yuanji Xu 
School: Hangzhou Foreign Languages School

Topic 2: Some Arithmetic Properties about the Factor of the Solution to Pell Equation and Its Applications in Diophantine Equations

Members: Yubo Zhao

Teacher: Baoguo Hao

School: The Affiliated High School of South China Normal University

Topic 3: Modeling and Planning of Snow Sweeping on Main Roads

Members: Yipeng Qin, Zhenyuan Gao, Cong Su

Teacher: Bin Cai

School: Suzhou High School of Jiangsu Province

\section{S.-T. Yau High School Mathematics Awards}

\section{Gold Award}

Topic: Coordinate-Free Characterization of Homogeneous Polynomials with Isolated Singularities

Members: Irene Chen

Teacher: Yau Stephen

School: Illinois Math and Science Academy

\section{Silver Award}

Topic: Research on the Combinatorial Transform Mathematics Problem Frog Leap

Members: Tian Zeng

Teacher: Bubin Tang

School: High School Affiliated to Renmin university of China

\section{Bronze Award}

Topic 1: Some Upper Bound Formula for Ramsey Numbers and Their Applications

Members: Zhiwei Xiang, Zhengzhen Qian, Shengbo Fu

Teacher: Lin Xia

School: Hangzhou Xizi Experimental Middle School

Topic 2: Geometric Model the Wonderful Tridimensional Kaleidoscope

Members: Zili Wang

Teacher: Cong Wu

School:Shenzhen High School

Topic 3: On the Upper Bound of Number Theoretic Function $\mathrm{Ff}(\mathrm{h})$

Members: Shenghan Lu

Teacher: Jinsong Li

School: High School Affiliated to Tsinghua University

\section{S.-T. Yau High School Mathematics Awards}

Gold Award

Topic: A Proof on the Non-differentiability of Weierstrass

Function in An Uncountable Dense Set

Members: Boyu Chen

Teacher: Rongsheng Jin

School: Shanghai Shibei High School

Silver Award

Topic: A Research On a Kind of Special Points Inside Convex

Members: Di Liu, Lun Yu

Teacher: Qingjie Yang

School: The High School Affiliated to China Renmin University

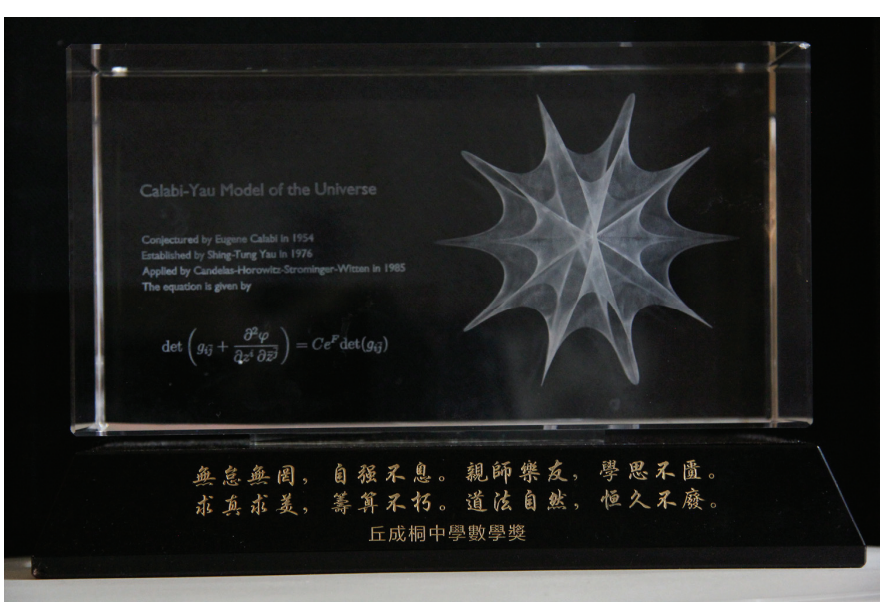

Trophy of S.-T. Yau High School Mathematics Silver Award

\section{Bronze Award}

Topic 1: From the Hinge Device to Curves of Linkages Members: Yue Gan, Yuge Chen, Lulu Sun

Teacher: Jie Jin

School: Zhengjiang Hangzhou No.2 High School

Topic 2: Extension, Enhancement and Analogue of the

Vasiliev Inequality

Members: Junxi Wu, Aolin Xiong, Zhe Liu

Teacher: Zhiming Yang

School: Guangdong Guangya High School

Topic 3: A New Definition of Distance for Graphs

Members: Farzan Vafa

Teacher: Yong Lin

School: Milton Academy, 180 Centre St., Milton, MA, USA 


\section{S.-T. Yau High School Applied Mathematical Sciences Awards}

Gold Award

Topic: The Study and Application of Velocity

Transmission in a Queue

Members: Taoyuanmin Zhu, Yunyi Zhang

Teacher: Jun Pan

School: Hangzhou Foreign Languages School

Silver Award

Topic: Understanding Flocking Dynamics in Nature

Members: Ang Li, Sung Lim, Qi Wang

Teacher: Chai Ming Huang

School: NUS High School of Mathematics and Science

Bronze Award

Topic 1: Actuarial Modeling on a Children's Protection Insurance

Members: Shu Zhang, Jing Li, Jianbin Li

Teacher: Lingshan Li

School: Jiangmen No.1 High School, Jiangmen, Guangdong

Topic 2: The Mathematical Model of the Oil Spilling in the

Gulf of Mexico Based on the Extended Fay Formulas

Members: Xiaoyang Li, Yu Cai

Teacher: Zhiming Yang

School: Guangdong Guangya High School

\section{S.-T. Yau High School Mathematics Awards}

Gold Award

Topic: Shock Profile for Gas Dynamics in Theemal

Nonequilibrium

Members: Xie Wang

Teacher: Luo Tao

School: Sidwell Friends Upper School, US

Silver Award

Topic: There Is a Non-Van Douwen Mad Family

Members: Zhuodong He

Teacher: Chengyu Zhang

School: Shenzhen Middle School

\section{Bronze Award}

Topic 1: The Relationship of The Slope to the Cutting Line of the Cubic Function

Members: Wenyuan Zeng

Teacher: Guoshuang Pan

School: Beijing National Day School

Topic 2: Strengthening proof of Bezout theorem

Members: Yukun Ding

Teacher: Wenhai Sun
School: Hefei No.168 Middle School

\section{S.-T. Yau High School Applied Mathematical Sciences Awards}

Silver Award

Topic: The Windpower Model of Typhoon on the South China Sea

Members: Yuxin Wan, Qianyun Hu

Teacher: Shishun Liu

School: ZhiXin High School of Guangzhou

Bronze Award

Topic 1: A Study of Domino and Its Applications Members: Jiaqi Yang, Tianchen $\mathrm{Xu}$, Chenyang Li

Teacher: Weifeng Li

School: Hangzhou Foreign Languages School

Topic 2: On Strongly Multiplicative Graphs

Members: Matthew Rauen

Teacher: Jesse Geneson

School: Pennsbury High School, USA

\section{S.-T. Yau High School Mathematics Awards}

Gold Award

Topic: On the Asymptotic Properties of the Analytical

Solutions to Two Functional Equations

Members: Chengyang Shao

Teacher: Lijun Yang

School: The High School Attached to Tsinghua University

Silver Award

Topic: A Diophantine Problem from Mathematical Physics Members: Zhi Ren, Dongchen Yang

Teacher: Lijiong Si

School: Hangzhou NO.2 High school of Zhejiang Province

Bronze Award

Topic 1: On a class of Discrete Maximum Value Problem Members: Yusheng Cai, Haizhou Zhao

Teacher: Chuanpeng Zhang

School: Hangzhou Foreign Language School

Topic 2: New Study on Identities Involving Euler Numbers and Bernoulli Numbers

Members: Ruibo Wei, Anjun Chu

Teacher: Junjie Zhang

School: Guangdong Experimental High School

Topic 3: The Structure and Topological Properties of

Möbius Strip Dissection

Members: Runzhu Fan, Xiang Li

Teacher: Suihai Luo 
School: The Affiliated High School of South China Normal University

\section{S.-T. Yau High School Applied Mathematical Sciences Awards}

\section{Silver Award}

Topic: The Optimal Pricing for Traffic Congestion

Members: Zongchan Chen, Nan Du

Teacher: Qiang Gong

School: Nanjing Foreign Language School

\section{Bronze Award}

Topic 1: A New Method to Measure and Compute Areas of Ellipses \& Volumes of Ellipsoids for Industrial Applications II

Members: Chenchao You, Han Tian

Teacher: Shengqiang Zhu

School: Nanjing Foreign Language School

Topic 2: Computationally Determining the Dimensions of the Homology Groups of Directed Graphs

Members: Ariya Shajii

Teacher: Gabor Lippner

School: Weston High School, MA

Topic 3: A Study on the Properties of CG and XD Figures Members: Zhijing Wang

Teacher: Qingming Yang

School: The High School Attached to Tsinghua University

\section{Hang Lung Mathematics Awards}

\section{Introduction}

The Hang Lung Mathematics Awards is a biennial mathematics research competition for secondary school students in Hong Kong. Founded in 2004 by Mr. Ronnie C. Chan, the Chairman of Hang Lung Properties, and Professor Shing-Tung Yau (Harvard University), the objective of the Awards is to encourage secondary students and teachers to realize their full creative potential in mathematics and science by stimulating their passion for intellectual discovery. Schools are invited to form teams of up to five students, and under the supervision of a teacher. Each team designs and carries out a mathematics research project, then submits a project report which summarizes the methodology, research, and results. The Scientific Committee evaluates the reports in a rigorous, multi-step review process that is similar to publishing an article in a scientific journal and determines teams to participate in the oral defense. The oral defense is modeled after the doctoral degree defense process of a brief public presentation followed by a closed-door inquiry. After the conclusion of the oral defense, the winners of the Hang Lung Mathematics Awards are decided and announced.
Hang Lung Properties and the Institute of Mathematical Sciences and Department of Mathematics at the Chinese University of Hong Kong are the co-organizers of the Hang Lung Mathematics Awards. At each competition, HK $\$ 1$ million is given out in monetary prizes in addition to tuition scholarships of HK\$374 thousands worth. There are eight Hang Lung Mathematics Awards: Gold, Silver, Bronze and up to five Honorable Mentions. Each award has four components: a Student Education Grant, a Teacher Leadership Award, a School Development Grant, and a Tuition Scholarship for a Master of Science (MSc) in Mathematics from the Chinese University of Hong Kong.

\section{Hang Lung Mathematics Awards Winners}

\section{Gold}

Topic: Marked Ruler as a Tool for Geometric

Constructions: From angle trisection to $n$-sided polygon

Members: Edward Sin Tsun Fan

School: Sha Tin Government School

Silver

Topic: Further Investigation on Buffon's needle problem Members: Fan Fan Lam, Ho Fung Tang, Ho Yin Poon, Lok Hin Yim, Yiu Tak Wong

School: Munsang College (Hong Kong Island)

\section{Bronze}

Topic: Ghost Leg

Members: Ting Fai Man, Hoi Kwan Lau, Shek Yeung, Man Kit Ho

School: Sha Tin Government Secondary School

\section{Hang Lung Mathematics Awards Winners}

\section{Gold}

Topic: How to Keep Water Cold: A Study about the Wet Contact Surface Area in Cylinder

Members: Cheuk Hin Cheng

Teacher: Mr. Kwok Tai Wong

School: S.K.H. Lam Woo Memorial Secondary School

\section{Silver}

Topic: On the Prime Number Theorem

Members: Yun Pui Tsoi

Teacher: Mr. Wai Man Ko

School: Shatin Government Secondary School 
Bronze

Topic: Construction of Tangents to Circles in Poincaré Model

Members: Fai Li, Chung Yam Li, Daniel Chung Sing Poon, King Ching Li

Teacher: Mr. Chun Yu Kwong

School: Wong Shiu Chi Secondary School

\section{Hang Lung Mathematics Awards Winners}

Gold

Topic: Isoareal and Isoperimetric Deformation of Curves Members: Kwok Chung Li, Chi Fai Ng

Teacher: Mr. Wing Kay Chang

School: Shatin Tsung Tsin Secondary School

Silver

Topic: Sufficient Condition of Weight-Balance Tree Members: Chi Yeung Lam, Yin Tat Lee

Teacher: Mr. Chun Kit Ho

School: The Methodist Church Hong Kong Wesley College

Bronze

Topic: Fermat Point Extension: Locus, Location, and Local Use

Members: Fung Ming Ng, Chi Chung Wan, Wai Kwun Kung, Ka Chun Hong

Teacher: Mr. Yiu Kwong Lau

School: Sheng Kung Hui Tsang Shiu Tim Secondary School

\section{Hang Lung Mathematics Awards Winners}

Gold

Topic: Expressibility of Cosines as Sum of Basis Members: Kwok Wing Tsoi, Ching Wong

Teacher: Mr. Yan Ching Chan

School: Po Leung Kuk Centenary Li Shiu Chung Memorial College

Silver

Topic: Curve Optimization Problem

Members: Ping Ngai Chung

Teacher: Ms. Mee Lin Luk

School: La Salle College

Bronze

Topic: Orchard Visibility Problem

Members: Trevor Chak Yin Cheung, Yin To Chui

Teacher: Mr. Kwok Kei Chang

School: Buddhist Sin Tak College

\section{Hang Lung Mathematics Awards Winners}

Gold

Topic: Towards Catalan's Conjecture

Members: Chung Hang Kwan

Teacher: Mr. Yat Ting Tong

School: Sir Ellis Kadoorie Secondary School (West Kowloon)

Silver

Topic: Cutting Twisted Solid Tori (TSTs)

Members: Yiu Shing Wong, Ho Yin Lau, Kai Lai Chan, Kai Shing Mok, Tsz Nam Chan

Teacher: Mr. Sai Hung Chan

School: Sha Tin Government Secondary School

Bronze

Topic: Complexity Reduction of Graphs

Members: Tsam Kiu Pun

Teacher: Mr. Cyril Lee

School: St. Mary's Canossian College

\section{S.-T. Yau High School Mathematics Awards in Taiwan}

In 2009, Professor Shing-Tung Yau initiated to hold an annual mathematics competition for high school students in Taiwan, aiming to promote intellectual discovery and to stimulate scientific creativity among the youth of Taiwan. This idea was made possible thanks to the generous support from Dr. Fan-cheng Tseng, the Vice Chairman of TSMC.

Each team shall consist of one student only and shall be led by a teacher of the same secondary school. The student must perform an independent study and may ask for opinions and accept guidance from the teacher. The research topic can be any problem from pure and applied mathematics.

Eight to twelve teams will compete in the final of S.-T. Yau High School Mathematics Awards. There will be:

1. No more than one Gold Award: 60,000NTD for each student and 30,000NTD for each teacher

2. No more than three Silver Awards: 40,000NTD for each student and 20,000NTD for each teacher

3. Honorable Mentions: 20,000NTD for each student and 10,000NTD for each teacher

4. Judge's Awards: 5,000NTD for each student

A school will receive Excellent Group Award if its students win more than two awards, at least one of which is Gold or Silver Award.

The awardee of Gold Award and Silver Award will receive scholarship up to 480,000 NTD and 240,000 NTD 
respectively during their studies in universities if they major in Mathematics or Applied Mathematics. A school will receive Excellent Group Award if its students win more than two awards, at least one of which is Gold or Silver Award.

The Award has excited young students for mathematical studies Many of participants choose to major in Mathematics at the end. The Award also has drawn lots of attention from the society. For example, the touching story of Che-Kuan Su appeared in all major newspapers and Medias the day after the ceremony.

\section{S.-T. Yau High School Mathematics Awards in Taiwan}

\section{Gold Award}

Topic: Shortest circumference of the inscribed polygon within a polygon

Member: Han-Ching Ou

School: National Wu-Ling Senior High School

\section{Silver Award}

Topic 1: Magic triangles

Member: Chi-Yun Hsu

School: Taipei First Girls High School

Topic 2: The Secretary Problem: Two-Player Extensions and Going Back

Member: Po-En Chen

School: National Experimental High School at Hsinchu Science Park

\section{S.-T. Yau High School Mathematics Awards in Taiwan}

\section{Gold Award}

Topic: The trail: a generalization of ellipse Member: Chin-Chieh $\mathrm{Hu}$

School: National Hsinchu Senior High School

\section{Silver Award}

Topic 1: A study and generalization of "Bulls and Cows" Member: Ping Li

School: Taipei First Girls High School
Topic 2: Mirror transformation of polygons Member: Yu-Hsuan Lin

School: Taipei Municipal Jianguo High School

\section{S.-T. Yau High School Mathematics Awards in Taiwan}

\section{Gold Award}

Topic: Beyond 3, 5, 7: a generalization of Pythagorean Triples

Member: Hsueh-I Chen

School: Taipei First Girls High School

\section{Silver Award}

Topic 1: A symmetry problem of elliptic differential operators in potential theory

Member: Yu-Ping Wang

School: Sacred Hearts High School

Topic 2: A study of the conic section by elementary geometry

Member: Wei-En Liao

School: National Taichung First Senior High School

Topic: 3 A chessboard coloring problem

Member: Chia-Chin Li

School: Taipei Municipal Jianguo High School

\section{S.-T. Yau High School Mathematics Awards in Taiwan}

Gold Award

Members: Che-Kuan Su

Teacher: Yun-Tung Hung

School: The Affiliated Senior High School of National Taiwan Normal University

Silver Award

Member: Mien Wang

Teacher: Fang-Ju Hsieh

School: National Experimental High School at Hsinchu Science Park

Member: Cheng-Min Chiang

Teacher: Peng-Yu Shen

School: Taipei Municipal Jianguo High School 